\title{
New Hybrid AD Methodology for Minimizing the Total Amount of Information Content: A Case Study of Rehabilitation Robot Design
}

Tao Yang $^{1^{*}} \mathbb{0}$, Xueshan $\mathrm{GaO}^{2,3}$ and Fuquan Dai ${ }^{4}$

\begin{abstract}
Converting customer needs into specific forms and providing consumers with services are crucial in product design. Currently, conversion is no longer difficult due to the development of modern technology, and various measures can be applied for product realization, thus increasing the complexity of analysis and evaluation in the design process. The focus of the design process has thus shifted from problem solving to minimizing the total amount of information content. This paper presents a New Hybrid Axiomatic Design (AD) Methodology based on iteratively matching and merging design parameters that meet the independence axiom and attribute constraints by applying trimming technology, the ideal final results, and technology evolution theory. The proposed method minimizes the total amount of information content and improves the design quality. Finally, a case study of a rehabilitation robot design for hemiplegic patients is presented. The results indicate that the iterative matching and merging of related attributes can minimize the total amount of information content, reduce the cost, and improve design efficiency. Additionally, evolutionary technology prediction can ensure product novelty and improve market competitiveness. The methodology provides an excellent way to design a new (or improved) product.
\end{abstract}

Keywords: Axiomatic design, Amount of information content, Trimming, Rehabilitation robot

\section{Introduction}

With the development of technology, an increasing number of technical measures have become available to solve engineering problems. Designers and engineers are beginning to realize that there are multiple alternative technological solutions (design parameters) for achieving specific needs (customer requirements). In the contemporary era characterized by the explosive development of technology, customer demands have become personalized and diversified, and emphasis has been placed on quality and the customer experience [1]. However, traditional research focused on finding appropriate technical solutions based on customer needs, organizing the design process, and solving practical engineering problems is hampered by information occlusion, the limited

\footnotetext{
*Correspondence: ayngtao@126.com

1 School of Design, Fujian University of Technology, Fuzhou 350118, China Full list of author information is available at the end of the article
}

knowledge and experience of engineers, and the lack of a systematic design philosophy for guiding the design process. In other words, designers were limited by technical factors, so they focused on the process of determining design parameters.

Suh [2] suggested that the information axiom could be used in optimal selection when multiple functional requirements (FRs) exist. Suh noted that one could minimize the information content by specifying the largest allowable tolerance when stating FRs and integrating the design parameters (DPs) into a single physical variable. Evaluation of design schemes through information axioms can optimize design schemes, increase the success rate of design and development, and enhance robustness [3-6].

In recent years, many scholars have performed extensive research to expand and improve AD theory. Emilio Sarno et al. [7] associated TRIZ and reliability-centred maintenance (RCM) with $A D$ to obtain practical 
solutions. Kremer et al. [8] presented an AD, TRIZ, and mixed integer programming (MIP) method to develop innovative designs. Ko [9] modelled an AD and TRIZ hybrid approach to solve contradictions.

Although these studies have successfully provided the basic principles and conceptual framework for product innovation design and improvement, most of these studies focused on the independence of axiomatic design, especially decoupling the design matrix if it is coupled. Most research on the information axiom has concentrated on the evaluation scheme. Krishnapillai and Zeid suggested that each designer has a unique way of generating design alternatives [10]. Each design alternative has advantages and disadvantages that affect customer satisfaction [11].

A novel systematic method is lacking that can help designers minimize the total amount of information content to create innovative products that can surprise customers in the context of new trends and competitive markets.

Suh [4] suggested that the information axiom can be used in optimal selection when multiple functional requirements exist. Suh noted that one could minimize the information content by specifying the largest allowable tolerance when stating FRs and integrating the design parameters into a single physical variable.

Although Suh proposed some guidelines for minimizing the total amount of information content, there is still a lack of specific practical technology for implementing the relevant work. The theory most similar to Suh's information axiom concept is trimming theory [12], which aims to remove or integrate parts and components while maintaining system functionality $[13,14]$. However, trimming theory does not provide specific methods for identifying the design parameters that should be trimmed, does not define information content, and lacks a theoretical framework.

Therefore, we can redefine the meaning of minimizing the total amount of information content, which involves not only reducing the number of information components, the uncertainty of design parameters and the complexity of consumer operations but also enhancing robustness. This paper attempts to provide a new design model to avoid unnecessary complexity in the design system and enhance customer satisfaction and help designers simultaneously solve problems and evaluate projects.

This paper introduces the AD methodology, trimming technology, ideal final results, and evolution theory in Section 2. Section 3 presents the proposed hybrid methodology. In Section 4, a case study is investigated to justify and verify the method. Finally, the conclusions are summarized in Section 5.

\section{Methodology}

To meet customer requirements and improve customer satisfaction, designers need to find the best designs among the alternative designs by continuously considering the principle, effect and structure of design parameters to achieve specific functions. In this process, to cope with fierce market competition, companies must improve the customer experience at the lowest cost, so the design process becomes complex. With developments in science and technology, there are now many ways to achieve specific functions and increase the amount of information processed. However, from the customer experience perspective, it is best to achieve these functions with as few operations as possible. The methodology described below was proposed to improve the traditional axiomatic design and minimize the total amount of information content under the premise of satisfying the independence axiom constraint based on AD theory, the trimming technique, the ideal final results (IFRs), and technology evolution theory.

\subsection{Axiomatic Design}

Suh proposed the AD concept in 1978 to maximize the productivity of a manufacturing system in all cases. Suh showed that when axiom conditions are satisfied, the robustness, performance, and reliability of the system and corresponding organization are improved.

Axiomatic design defines the process of product design as a procession of the designer choosing appropriate design parameters (DP) to achieve specific requirements. The main theoretical framework involves mapping relationships among customers, requirements and design parameters. The process includes 4 steps: (1) abstracting the customer needs (CNs); (2) mapping the customer needs in the FR space; (3) constructing the product function structural tree and determining design parameters to achieve specific functions based on the relevant principle, effect and structure (e.g., design parameters; DPs); and (4) mapping the selected optimal solution to the production process (with process variables; PVs). Finally, a product is obtained. According to the axiomatic principle, the mapping process and areas are shown in Figure 1.

In addition to the main theoretical framework, there are two design axioms for product design, which can be stated as follows.

Axiom 1 (the independence axiom) involves maintaining the functional requirements of independence. Specifically, when there are two or more functional requirements, every requirement should be met without affecting the others, which requires the selection of design parameters that meet the overall functional requirements as well as the individual functional 


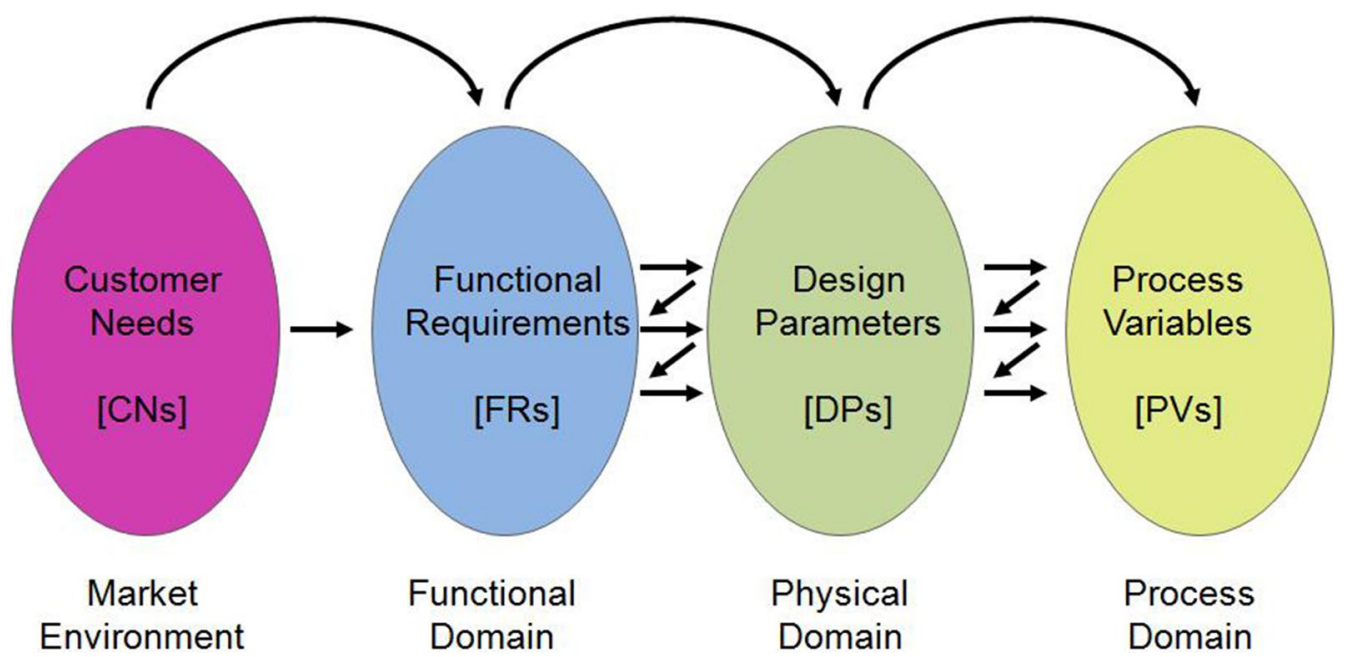

Figure 1 Mapping processes of domain sand areas (Suh, 1990)

requirements. When a DP changes, the corresponding FR changers and the other dependent FRs are affected.

Axiom 2 (the information axiom) involves minimizing the total amount of information content to obtain the conditions of the optimal scheme.

\subsection{Trimming Technique}

The trimming technique was first proposed by Litvin and Gerasimov in the mid-1990s and was first published in Russian and then in English [12]. Sheu and Ho developed a set of trimming methods with significant results [14].

Trimming is a method of deleting some components while enhancing system ideality and reducing costs [14]. Trimming can eliminate or reduce harmful effects on the system, improve maintainability, reduce the difficulty of operations, and reduce the system costs. These traits are all necessary in contemporary design. There are many complex products, and customers usually need to read complicated manuals and follow specific functions and steps. These steps can be simplified or avoided with trimming methods. Additionally, trimming can be used to minimize the total amount of information content.

\subsection{Ideal Final Result}

Obtaining the ideal final result (IFR) $[15,16]$ is one step in the TRIZ [17-19]. The following formula can express the IFR.

$$
\text { Ideality }=\frac{\sum \text { Benefits }}{\sum \text { Cost }+ \text { Harmful function }} .
$$

The ideal solution originally proposed by Altshuller is that the system develops in the direction of improving the ideal level of the system. Moreover, the IFRs achieve the most significant degree of self-service (self-realization, self-delivery, and self-control) in the case of a minimal change in the system.

This theory can help improve the existing technical system by considering the solution to the problem and obtaining an optimal solution by overcoming traditional boundaries and seeking new technical methods.

\subsection{Technology Evolution Theory}

Technology evolution theory is an essential branch of TRIZ. Altshuller suggested that the evolution of a technological system is not random but follows specific objective laws $[18,19]$. Many scholars have since enriched technology evolution theory [20,21]. The eight patterns of evolution proposed by Terninko et al. [22] were considered in the paper.

\section{New Hybrid Axiomatic Design Methodology}

The prerequisite for minimizing information is to determine the design parameters or functions required by a given operation. We first use AD theory to map functional requirements and design parameters for structural attribute correlation calculations. First, AD is adopted to analyse the design problem, decompose the main problem into a sub-hierarchy level problem and map design parameters. Then, the attributes of the design parameters are determined to be related or not, which is the 
core objective and key step in information minimization. We propose the following criteria for attribute-related judgement.

(1) Determining whether functional requirements (FRs) are relevant or not.

The goal of this criterion is to determine whether some FRs can be achieved with a single design parameter (DP) based on an achievable index.

(2) Determining whether design parameters (DPs) are relevant or not.

The objective of this criterion is to determine whether some components can be replaced with an intrinsic function or other components based on an achievable index.

(3) Calculating the total amount of information content.

$$
I=\log _{2} 1 / p
$$

where $I$ is the total amount of information content and $p$ is probability.

The design parameters to which attributes are related should be iteratively matched and merged based on trimming techniques, the IFR, or technology evolution theory. After the above operations, we obtain the new design parameters and a new solution and then determine whether the new solution has the lowest information content. If not, the physical domain (PD) is transformed back to the functional domain (FD) based on zigzagging, which is the process of decomposing a design into hierarchies by alternating between domains and redescribing the design parameters as functional requirements. Finally, this process is repeated until the optimal solution is obtained.

An operational model is constructed to help designers conveniently use the proposed method in product design, as shown in Figure 2 (we propose a hybrid algorithm that includes mapping, zigzagging, and iterative matching and merging based on DPs, as shown in Appendix).

\section{Case Study}

\subsection{Defining the Design Problems}

Many regions have ageing societies, and there are many people with ambulatory dysfunction in those regions. Moreover, many areas are facing shortages of medical workers [23]. A rehabilitation robot is regarded as one way to solve this problem [24, 25]. We worked with the China Rehabilitation Research Center (CRRC) to design a new mobile chaperonage lower limb rehabilitation training robot (MCLLRTR). The requirements of the robot are summarized as follows:

(1) Assist impaired individuals in exercises for leg rehabilitation;

(2) Detect and record the gait and recovery information;

(3) Provide a flexible mechanical structure for patient security;

(4) Have as few operational rules as possible for the lower limbs;

(5) Have a low cost.

\subsection{Innovative Process Based on the New Hybrid Methodology}

Step 1: Define the necessary level of AD syntax.

According to the CRRC, the training content used in rehabilitation varies according to the level of dysfunction and physical condition. In this work, we mainly focused on late-stage rehabilitation training and the development of a mobile robot for rehabilitation training. The essential FR $\left(\mathrm{FR}_{1}\right)$ can be expressed as follows: the design parameters must be appropriate for a lower limb rehabilitation training robot.

An appropriate design parameter $\left(\mathrm{DP}_{1}\right)$ is required to satisfy $\mathrm{FR}_{1}$. Thus, $\mathrm{DP}_{1}$ can be described based on the relevant conversion technology and structure.

There is only one design parameter $\left(\mathrm{DP}_{1}\right)$, and there is no property-related phenomenon. Therefore, we need to decompose FR and DP continuously.

According to $\mathrm{DP}_{1}$, the further decomposition of $\mathrm{FR}_{1}$ represents the following FRs:

$\mathrm{FR}_{11}$ : Provides a proper rehabilitation mechanism for rehabilitation training involving hemiplegic patients;

$\mathrm{FR}_{12}$ : Able to detect and record gait and fall information;

$\mathrm{FR}_{13}$ : Able to adjust dimensional parameters and ensure safety;

$\mathrm{FR}_{14}$ : Provide simple operations with good interactivity; and

$\mathrm{FR}_{15}$ : Have a low cost. 


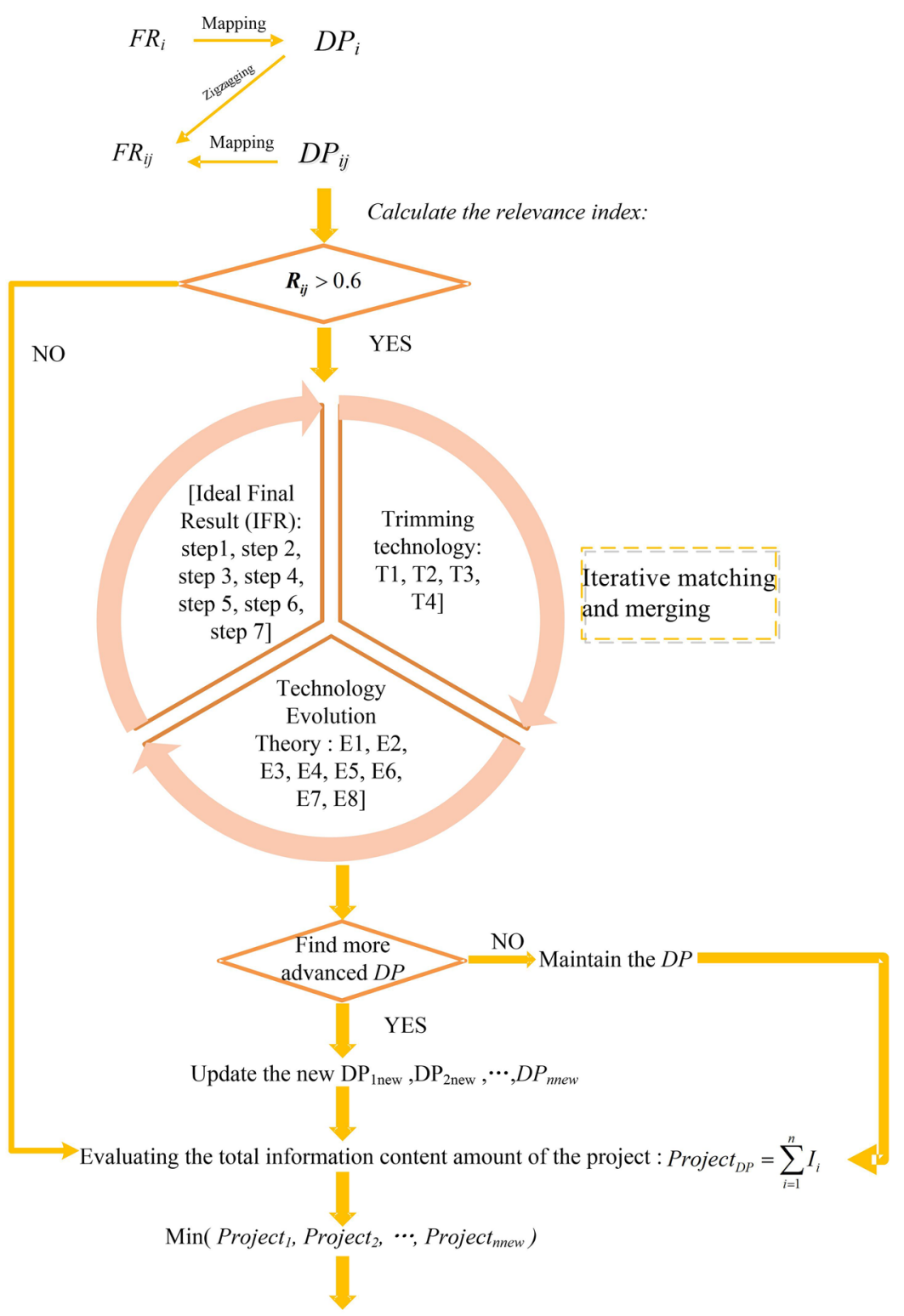

Obtain the $D P$ that has the lowest information content

Where:

step 1 . What is the ultimate goal of the system? step 3. What are the barriers to IFR?

step 5 . What is the condition of this barrier?

step 7. what resources can be used in the super system and environment?

$\mathrm{T} 1=$ Implementation of other components or systems in the system,

$\mathrm{T} 2=$ Achieving their functions through the components themselves,

$\mathrm{T} 3=$ Deleting the original components to achieve the function,

T4=Removing the original function of the role of components.

E1=Evolution in stages (or according the S-curve), E2= Evolution toward increased ideality,

$\mathrm{E} 3=$ Non uniform development of system elements, E4=Evolution toward increased dynamism and controllability,

E5=Increased complexity then simplification, $\quad$ E6=Evolution with matching and mismatching components,

E7 = Evolution toward micro level and increased use of fields,

E8=Evolution toward decreased human involvement

Figure 2 Operational model for minimizing the total amount of information content 
The FR hierarchy is constructed from the corresponding DP hierarchy.

The alternative solutions and various DPs are analysed based on the new hybrid methodology, as previously noted.

Step 2: Calculate the relevance index of each domain, $i$ and $j$, to determine the design parameters to which attributes are related.

The final relevance index for each domain, $i$ and $j$, is calculated based on the accumulation of weighted subrelevance indices from the abovementioned 3 criteria indices using Eq. (3).

$$
R_{i j}=\omega_{F} F_{i j}+\omega_{D} D_{i j}+\omega_{P} P_{i j},
$$

where $i$ and $j$ are the indices of the relevance calculation and $R_{i j}$ is the final relevance index for DPs $i$ and $j$, and its value ranges from -1 to $1 . F_{i j}, D_{i j}$, and $P_{i j}$ are the three relationship indices for $i$ and $j$. These indices are related to the functional requirements, design parameters, and properties. $\omega_{F}, \omega_{D}$, and $\omega_{P}$ are the weights of the corresponding 3 indices. The weight of each factor is between 0 and 1 , and the sum of the 3 weights is equal to 1 .

Step 3: Iteratively match and merge the design parameters until the DP with the lowest information content is obtained.

We can identify the target DPs (according to the following iteration condition: if $\left.R_{i j}>0.6\right)$ that can be integrated or trimmed based on the relevance index and then iteratively combine them. According to $\mathrm{FR}_{12}$, the corresponding $\mathrm{DP}_{12}$ should be able to detect gait and fall information and record the training time, number of steps, and movement speed. We conducted related investigations and research on the existing gait detection technology and summarized several common methods, such as those based on image detection [26], environmental perception-based detection [27], and wearable sensor detection [28, 29].

We can use the hybrid model to minimize the total amount of information content. We identified $D P_{12}$ as the target of iterative combination based on the relevance index. According to trimming technique (1), as discussed in Section 2.2, the IFRs (selfrealization) and technology evolution theory (in this case, evolution rule 5: transition to a flexible system or a mobile system to improve controllability), as noted in Sections 2.3 and 2.4, we finally met some detection requirements by arranging the photoelectric sensors at specific spatial locations and applying a novel classifier algorithm. The novel classifier algorithm is detailed in another paper [30-32]. Compared to other sensor combination methods, the use of photoelectric sensors dramatically reduces the complexity of the design. We can list the design parameters that meet the functional requirements in the design matrix and calculate the information content of the design parameters. The relevance index and total amount of information content between the FR and DP matrices are given in Table 1 based on the new hybrid methodology, as previously discussed.

In this article, we use the accuracy rate of different alternative solutions as the basis for calculating the information content and set the recognition accuracy rate $A_{i}$ to calculate the information content using Eq. (4):

$$
A_{i}=\frac{T P+T N}{P+N},
$$

where $T P+F N$ represents the number of samples that are positive, and $P+N$ is the total number of samples.

$$
\begin{aligned}
P_{i} & =A_{i}, \\
I_{i} & =\log _{2} \frac{1}{P_{i}} .
\end{aligned}
$$

As shown in Table 1, we found that for gait detection and fall detection, the solution of wearable sensors has the least information content; for step counting, detecting the training time and detecting the patient's walking speed, the solution of photoelectric sensors has the least information content.

To accurately recognize the various states of the patient in real time, we proposed a multi-sensor system to obtain multiple features and classify activities from different dimensions. The system board was designed with a STM32 microprocessor powered by a $5 \mathrm{~V}$ battery. To improve the accuracy of fall detection, we developed a tri-sensor detection system (as shown in Figure 3) for our specific rehabilitation robot. The photoelectric sensors collect the spatial distribution features of the gait for activity recognition. The tension sensors collect the directional features by sampling the difference in the same-side sensor signals. 
Table 1 Information content analysis based on the new hybrid axiomatic design methodology

\begin{tabular}{|c|c|c|c|c|c|c|c|}
\hline & \multicolumn{7}{|l|}{$\mathrm{DP}_{12}$} \\
\hline & \multirow{2}{*}{$\begin{array}{l}\text { Alternative } \\
\text { solutions }\end{array}$} & \multicolumn{5}{|c|}{ The final relevance index } & \multirow{2}{*}{$\begin{array}{l}\text { Information content } \\
\text { (Recognition } \\
\text { accuracy) }\end{array}$} \\
\hline & & $\begin{array}{l}\text { Image } \\
\text { processing }\end{array}$ & $\begin{array}{l}\text { Wearable } \\
\text { sensor }\end{array}$ & $\begin{array}{l}\text { Photo- } \\
\text { electric } \\
\text { sensor }\end{array}$ & $\begin{array}{l}\text { Surrounding } \\
\text { environment } \\
\text { perception }\end{array}$ & Tension sensor & \\
\hline \multicolumn{8}{|l|}{$\mathrm{FR}_{12}$} \\
\hline \multirow[t]{3}{*}{$\mathrm{FR}_{121}$ : Gait detection } & Image processing & $x$ & 0.54 & 0.35 & & & 0.234 \\
\hline & Wearable sensor & & $x$ & 0.68 & & & 0.136 \\
\hline & Photoelectric sensor & & & $x$ & & & 0.322 \\
\hline \multirow[t]{5}{*}{$\mathrm{FR}_{122}$ : Fall detection } & Image processing & $x$ & 0.5 & -1 & 0.42 & 0.62 & 0.12 \\
\hline & Wearable sensor & & $x$ & -1 & 0.45 & 0.82 & 0.089 \\
\hline & Photoelectric sensor & & & $x$ & -1 & 0.82 & 0.415 \\
\hline & $\begin{array}{l}\text { Surrounding environ- } \\
\text { ment perception }\end{array}$ & & & & $x$ & 0.35 & 0.252 \\
\hline & Tension sensor & & & & & $x$ & 0.184 \\
\hline \multirow{4}{*}{$\begin{array}{l}\mathrm{FR}_{123} \text { : Detecting the } \\
\text { training time }\end{array}$} & Image processing & $x$ & 0.42 & -1 & -1 & & 0.252 \\
\hline & Wearable sensor & & $x$ & 0.42 & 0.3 & & 0.029 \\
\hline & Photoelectric sensor & & & $x$ & -1 & & 0.007 \\
\hline & $\begin{array}{l}\text { Surrounding environ- } \\
\text { ment perception }\end{array}$ & & & & $x$ & & 0.515 \\
\hline \multirow{4}{*}{$\begin{array}{l}\mathrm{FR}_{124} \text { : Detecting the } \\
\text { number of steps }\end{array}$} & Image processing & $x$ & 0.42 & 0.56 & -1 & & 0.515 \\
\hline & Wearable sensor & & $x$ & 0.78 & 0.76 & & 0.089 \\
\hline & Photoelectric sensor & & & $x$ & 0.64 & & 0.007 \\
\hline & $\begin{array}{l}\text { Surrounding environ- } \\
\text { ment perception }\end{array}$ & & & & $x$ & & 2.32 \\
\hline \multirow{4}{*}{$\begin{array}{l}\mathrm{FR}_{125} \text { : Detecting the } \\
\text { patient's walking } \\
\text { speed }\end{array}$} & Image processing & $x$ & 0.42 & 0.52 & 0.32 & & 0.252 \\
\hline & Wearable sensor & & $x$ & 0.42 & 0.3 & & 0.515 \\
\hline & Photoelectric sensor & & & $x$ & 0.25 & & 0.007 \\
\hline & $\begin{array}{l}\text { Surrounding environ- } \\
\text { ment perception }\end{array}$ & & & & $x$ & & 2.32 \\
\hline
\end{tabular}
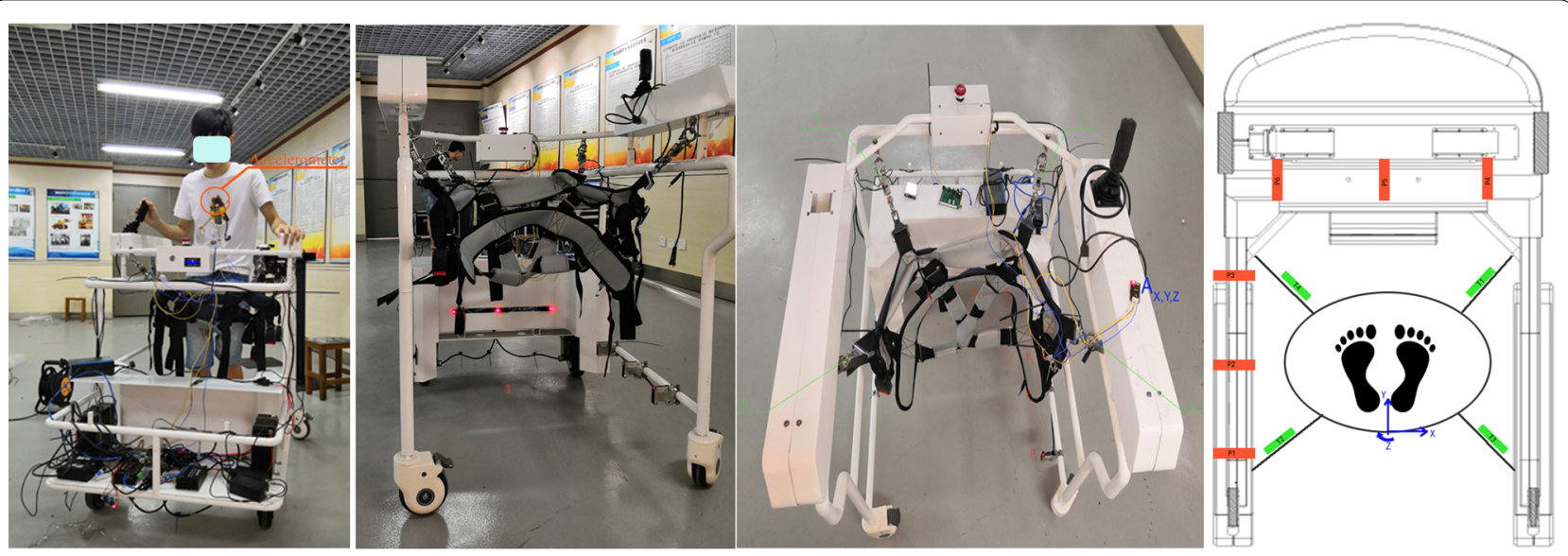

Figure 3 The tri-sensor detection system 
The accelerometer sensor collects kinematic information for activity recognition.

Through the new hybrid methodology, we successfully designed a new low-cost robot that meets almost all the relevant requirements. Compared to the previous rehabilitation training robots, the cost of the new rehabilitation robot is reduced by as many as $42 \%$, and it allows patients to achieve omnidirectional actuation with straightforward manipulation, which significantly improves patient satisfaction.

\section{Conclusions}

(1) Previous work has documented the effectiveness and strength of $\mathrm{AD}$ in the process of product design. Moreover, many scholars have performed extensive research to enrich and perfect the related theory. However, these studies did not focus on minimizing the total amount of information content, which is important for improving system ideality and customer experience. In this study, we proposed an approach for minimizing the total amount of information content based on trimming technology, IFRs, and technology evolution theory. The method can designers generate technically better solutions and create innovative products that can surprise users considering new trends and competitive markets.

(2) This approach consists of an operational model of new hybrid methodology and related algorithms. Through the grammar structure of AD theory, the hierarchy of FRs is decomposed and mapped to the DPs to determine the design parameters for which attributes are related based on the relevance index. Finally, we reduce the total amount of information content by iteratively matching and merging the design parameters that meet the independence axiom constraint, and the attributes are related based on trimming technology, the IFRs, and technology evolution theory. The operational model can help designers simultaneously solve problems and evaluate projects.

(3) Notably, to the best of our knowledge, this is the first study to propose a new hybrid methodology based on the iterative matching and merging of design parameters to minimize the total amount of information content. Our results provide compelling evidence for the effectiveness of this approach. However, some limitations are worth noting. The iterative matching and merging process is time intensive, and some professional knowledge is required. Future work should focus on developing an effective control method for the iterative process.

\section{Acknowledgements}

The authors sincerely thanks to Doctor Yue Lin of IWINTALL.INC for his critical discussion and reading during manuscript preparation.

\section{Authors' Contributions}

TY was in charge of the whole trial; TY wrote the manuscript; XG and FD assisted with sampling and laboratory analyses. All authors read and approved the final manuscript.

\section{Authors' Information}

Tao Yang, born in 1981. He received his PhD degree from Beijing Institute of Technology, China, in 2020. He is with School of Design, Fujian University of Technology, China. His research interests include industry design, man-machine system and rehabilitation robotics.

Xueshan Gao, born in 1966, is currently a professor and a PhD candidate supervisor at Beijing Institute of Technology, China. His research interests include mobile robot, medical robot. He is a senior member of Chinese mechanical Engineering Society (CMES) and a member of IEEE.

Fuquan Dai, born in 1987. He received his B.S. degree and PhD degree in engineering from Department of Microelectronics, Beijing Institute of Technology, China, in 2009, and 2015, respectively. From 2015 to now, he is with Department of Mechanical and Automotive Engineering, Fujian University of Technology, China. His research interest includes control theory that utilizes dynamics of physical systems, and robotics.

\section{Funding}

Supported by Research Startup Fund Project of Fujian University of Technology (Grant No. GY-Z20089), Science Foundation for Young Scholars of Fujian Province of China (Grant No. 2018J05099) and Education and Scientific Research Projects of Young Teachers in Fujian Province of China (Grant No. JAT160313).

\section{Competing Interests}

The authors declare no competing financial interests.

\section{Author Details}

${ }^{1}$ School of Design, Fujian University of Technology, Fuzhou 350118, China.

${ }^{2}$ School of Mechatronical Engineering, Beijing Institute of Technology, Beijing 100118, China. ${ }^{3}$ Department of Mechanical and Automotive Engineering, Fujian University of Technology, Fuzhou 350118, China. ${ }^{4}$ Fujian Haiyuan Composite Materials Technology Co., Ltd., Fuzhou, China. 


\section{Appendix}

appendix 1 Algorithm for the iterative matching and merging of related $D P s$ to generate $D P_{n e w}$

$<$ Design problem ID\#>

Step 1: Functional requirements

While (the mapping $D P$ does not meet the requirement of $F R$ )

Subfunctions: $\{[$ convert-revert | couple-separate $\mid \quad]\} F R_{1}, F R_{2}, \ldots, \mathrm{F} R_{n}$

Mapping to design parameters: $D P_{1}, D P_{2}, \ldots, D P_{n}$;

Zigzagging $D P s$ back to the functional domain $(F R)$ and dividing $F R$ into sub-functions $F R_{1 l}$,

$$
F R_{12} \ldots F R_{n j}
$$

Mapping to design parameters: $D P_{11}, D P_{12}, \ldots, D P_{n j}$;

Loop

While (satisfy the independence axiom)

\{

for $i=1,2, \ldots, n$;

Compare $D P_{1}, D P_{n}$;

Compare $D P_{11}, D P_{n n}$;

Step 2: $\quad$ Calculate the relevance index: $R_{i j}=\omega_{F} F_{i j}+\omega_{D} D_{i j}+\omega_{P} P_{i j}$;

Step 3: $\quad$ Do (attributes are related (if $\boldsymbol{R}_{i j}>0.6$ )

\{

Ideal Final Result (IFR): step 1. What is the ultimate goal of the system? step 2. What is the idealized final result? step 3. What are the barriers to IFR? step 4. What is the result of this barrier? step 5. What is the condition of this barrier? step 6 . What resources can be used in the system? step 7. what resources can be used in the super system and environment?]; search for the IFR that can satisfy the requirement and maintain the functional independence axiom constraint.

If (There is an ideal final result)

\{Update the new $D P_{\text {new; }}$ Break\}

Else

\{Maintain the $D P$ \}

Trimming technology: T1=Implementation of other components or systems in the system, $\mathrm{T} 2=$ Achieving their functions through the components themselves, $\mathrm{T} 3=$ Deleting the original components to achieve the function, T4=Removing the original function of the role of components.]; \%Analyse the DPs to trim the components that can be merged while maintaining the overall functionality and independence axiom constraint.

If (Some DPs can be merged)

$\left\{\right.$ Update the new $D P_{n e w} ;$ Break

Else

\{Maintain the $D P s\}$ \}

Technology Evolution Theory: E1=Evolution in stages (or according the S-curve), E2= Evolution toward increased ideality, E3=Non uniform development of system elements, E4=Evolution toward increased dynamism and controllability, E5=Increased complexity then simplification (reduction), Evolution with matching and mismatching components, E7= Evolution toward micro level and increased use of fields, E8=Evolution toward decreased human involvement]; \%Search for more advanced $D P$ s that meet the total functionality and independence axiom constraints.

If (Find more advanced $D P S$ )

\{Update new $D P_{\text {new }} ;$ Break\}

Else

$\{$ Maintain the $D P s\}$

\}

$\left\{D P_{1}, D P_{2}, \ldots, D P_{\text {lnew }} D P_{2 \text { new }}, \ldots, D P_{\text {inew }}\right\}$

Evaluate the information content of the project that contains the $D P \mathrm{~s}$ :

$$
\text { Project }_{D P}=\sum_{i=1}^{n} \boldsymbol{I}_{\boldsymbol{i}} \quad \% \text { Total information content of Project } i
$$

Min (project 1 , project $2, \ldots$, project ${ }_{D P_{\text {inew }}}$ );

Obtain the $D P$ that has the lowest information content. 
Received: 22 October 2019 Revised: 10 November 2020 Accepted: 12 November 2020

Published online: 26 November 2020

\section{References}

[1] M Kuniavsky. Observing the customer experience: a practitioners guide to customer research. San Francisco, USA: Morgan Kaufmann Publishers, 2003

[2] N P Suh. Axiomatic design-Advances and applications. New York: Oxford University Press, 2001.

[3] Martin G Helander, Lin Li. Axiomatc design in ergonomics and an extension of the information axiom. Journal of Engineering Design, 2002, 13(4): 321-339.

[4] N P Suh. Axiomatic design theory for systems. Research in Engineering Design, 1998, 10: 189-209.

[5] Osman Kulak, Cengiz Kahraman. Fuzzy multi-attribute selection among transportation companies using axiomatic design and analytic hierarchy process. Information Science, 2005, 170: 191-210.

[6] Tichun Wang, Bingfa Chen, Liangfeng Bu. Multi-attribute optimal selection model of large-scale hydraulic turbine scheme design based on information axiom. Journal of Nanjing University of Aeronautics \& Astronautics, 2011, 43(6): 822-826.

[7] Emilio Sarno, Vipin Kumar, Wei Li. A hybrid methodology for enhancing reliability for large systems in conceptual design and its application to the design of a multiphase flow station. Research in Engineering Design, 2005, 16: 27-41.

[8] G O Kremer, M C Chiu, C Y Lin, et al. Application of axiomatic design, TRIZ, and mixed integer programming to develop innovative designs: Alocomotive ballast arrangement case study. The International Journal of Advanced Manufacturing Technology, 2012, 61(5-8): 827-842.

[9] Yao-Tsung Ko. Modeling a hybrid-compact design matrix for new product innovation. Computer \& Industrial Engineering, 2017, 107: 345-359.

[10] R Krishnapillai, A Zeid. Mapping product design specification for mass customization. Journal of Intelligent Manufacturing, 2006, 17(1): 29-43.

[11] Prabhat Kumar, Puneet Tandon. A paradigm for customer-driven product design approach using extended axiomatic design. Journal of Intelligent Manufacturing, 2019, 30(2): 589-603.

[12] V M Gerasimov, S S Litvin. Osnovnye polozheniya metodiki provedeniya funktsionalno-stoimostnogo analiza. Metodicheskiye rekomendatsyi. TRIZ Journal, 1992, 3.2. (in Russian)

[13] Q Li, G Cao, H Guo, et al. Product integrated innovation based on function. IFIP Advances in Information and Communication Technology, 2009, 304: 59-69.

[14] D D Sheu, M Hou. TRIZ-based systematic device trimming method: theory and applications. The International Journal of Systematic Innovation, 2012, 2(1): 2-21

[15] D Daniel Sheu, Ming-Chuan Chiu, Dimitri Cayard. The 7 pillars of TRIZ philosophies. Computer \& Industrial Engineering, 2020. 146: 106572

[16] Czesław Cempel. The ideal final result and contradiction matrix for machine condition monitoring with TRIZ. 5th International Congress of Technical Diagnostics, 2014, 588: 276-280.
[17] G Altshuler. Creativity as an exact science: The theory of the solution of inventive problems. Amsterdam: Gordon and Breach, 1984.

[18] G Altshuler. And suddenly the inventor appeared TRIZ the theory of inventive problem solving. 2nd ed. Amsterdam: Gordon and Breach, 1996.

[19] G Altshuller. Creativity as an exact science, Theory of Inventive problems solving. Moscow: Sovetskoye Radio, 1979.

[20] Jesús Delgado-Maciel, Guillermo Cortés-Robles, CuauhtémocSánchezRamírez, et al. The evaluation of conceptual design through dynamic simulation: A proposal based on TRIZ and system dynamics. Computer \& Industrial Engineering, 2020, 149: 106785.

[21] Muhammad Mansoor, Norman Mariun, Noor IzzriAbdulWahab. Innovating problem solving for sustainable green roofs: Potential usage of TRIZ-Theory of inventive problem solving. Ecological Engineering, 2017, 99 : 209-221.

[22] J Terninko, A Zusman, B Zlotin. Systematic innovation: an introduction to TRIZ. Boca Raton: CRC Press LLC, 1998.

[23] Syrine Soufi, Stéphane Chabrier, Laurent Bertoletti, et al. Lived experience of having a child with stroke: A qualitative study. European Journal of Paediatric Neurology, 2017, 21(3): 542-548.

[24] Quan Liu, Jie Zuo, ChangZhu, et al. Design and control of soft rehabilitation robots actuated by pneumatic muscles: State of the art. Future Generation Computer Systems, 2020, 113: 620-634.

[25] Di Shi, Wuxiang Zhang, Wei Zhang, et al. Assist-as-needed attitude control in three-dimensional space for robotic rehabilitation. Mechanism and Machine Theory, 2020, 154: 104044.

[26] B Mirmahboub, S Samavi, N Karimi, et al. Automatic monocular system for human fall detection based on variations in silhouette area. IEEE Transactions on Biomedical Engineering, 2013, 60(2): 427-436.

[27] D Litvak, Y Zigel, I Gannot. Fall detection of elderly through floor vibrations and sound. Engineering in Medicine and Biology Society. Proceedings of IEEE 30th Annual International Conference on Engineering in Medicine and Biology Society, Vancouver, BC, 2008: 4632-4635.

[28] Yi-Cho Fang, Ren-Jye Dzeng. Accelerometer-based fall-portent detection algorithm for construction tiling operation. Automation in Construction, 2017, 84: 214-230

[29] Lin Chen, Rong Li, Hang Zhang, et al. Intelligent fall detection method based on accelerometer data from a wrist-worn smart watch. Measurement, 2019, 140: 215-226.

[30] Tao Yang, Xueshan Gao, Jinmin Peng, et al. A novel activity recognition system for alternative control strategies of a lower limb rehabilitation robot. Applied Sciences, 2019, 9(19): 1-19.

[31] Tao Yang, Xueshan Gao. Adaptive neural sliding-mode controller for alternabtive control strategies in lower limb rehabilitation. IEEE Transactions on Neural Systems and Rehabilitation Engineering, 2020, 28(1): 238-247.

[32] Xueshan Gao, Tao Yang, Jinmin Peng. Logic-enhanced adaptive networkbased fuzzy classifier for fall recognition in rehabilitation. IEEE Access, 2020, 8(1): 57105-57113.

\section{Submit your manuscript to a SpringerOpen ${ }^{\oplus}$ journal and benefit from:}

- Convenient online submission

- Rigorous peer review

- Open access: articles freely available online

- High visibility within the field

Retaining the copyright to your article

Submit your next manuscript at springeropen.com 